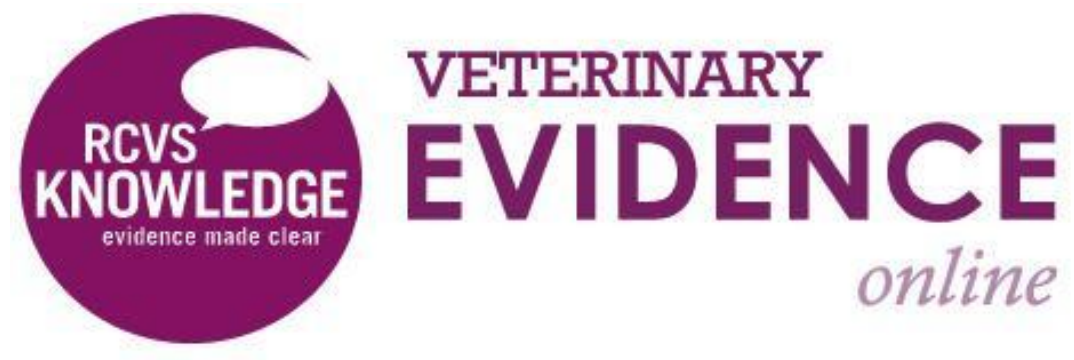

\title{
An Exploratory Study Investigating the Non- Clinical Benefits of Evidence-Based Veterinary Medicine
}

Elizabeth L. Jackson BAg (Hons), MBA, PhD, SFHEA ${ }^{1^{*}}$

Sarah Hauser BSc (Hons), MPA, MPP ${ }^{{ }^{*}}$

\footnotetext{
${ }^{1}$ The Royal Veterinary College, Hawkshead Lane, North Mymms, Hatfield, Hertfordshire, AL9 7TA, United Kingdom

Corresponding Author (eljackson@rvc.ac.uk)
}

ISSN: 2396-9776

Published: 30 May 2017

in: Vol 2, Issue 2

DOI: http://dx.doi.org/10.18849/ve.v2i2.97

Reviewed by: Dr Sebastian Arlt and Virginia Fajt (DVM, PhD, DACVCP) 
Objective: As little prior research exists about the non-clinical benefits of evidence-based veterinary medicine (EBVM), this exploratory study was conducted to identify non-clinical benefits of EBVM to veterinary practices, as well as highlighting the barriers to further implementation, and ways to overcome them.

Background: A PICO-based literature review (Hauser and Jackson, 2016) was conducted to establish current knowledge about the non-clinical benefits of EBVM. It found that while there are some papers suggesting a link between the practice of EBVM and better non-clinical benefits such as client satisfaction and client retention, a single study, focusing on the non-clinical benefits of EBVM, had yet to be conducted.

Evidentiary value: This exploratory study provides a solid basis for the further development of a confirmatory study of the themes identified in the interviews. The impact on practice from our findings is significant as it details the key areas where the use of EBVM can yield commercial benefits from the perspective of a group of EBVM experts via interview. It is entirely possible that international veterinary environments which mirror that of the UK will find this research beneficial.

Methods: Due to the paucity of data about the non-clinical benefits of EBVM, an exploratory, qualitative approach was taken to this research in order to build a platform for further confirmatory, quantitative investigation (Zikmund, 2003). In February and March 2016 interviews with 16 RCVS Knowledge Group chairs[1] were conducted. The interview guide contained broad, open-ended questions to explore existing tacit knowledge about the non-commercial benefits of EBVM. The interviews were audio recorded and transcribed verbatim and subsequently analysed using NVivo 11 software.

Results: This qualitative enquiry showed that the key areas where the use of EBVM can yield non-clinical benefits are through increased client satisfaction and retention, improved reputation and confidence of the veterinarian, as well as employee engagement. In order to yield these benefits the two main barriers, time and resources, need to be overcome.

Conclusion: The themes highlighted in this paper provide a solid, real world base for further, quantitative study of the non-clinical benefits of practising EBVM.

Application: The results of this research are applicable to practicing veterinarians, nurses and other practice staff. It is clear that practising EBVM not only leads to better clinical outcomes, but can also result in commercial benefits, such as better client retention and employee engagement

[1] Chairs of RVCS Knowledge Groups are recognised leaders in the field of EBVM and hold these invited positions within the UK's Royal College of Veterinary Surgeons.

\section{INTRODUCTION}

Little research exists on the non-clinical benefits of EBVM. While the clinical benefits have been fairly well documented, the business aspect of evidence-based veterinary medicine has not. In a situation such as this, Zikmund (2003) suggests an exploratory approach to help inform a more rigorous, conclusive study when there is a need for generating new ideas. Exploratory research is often qualitative in nature and thus does not attempt to provide conclusive answers to research questions. Rather, it allows researchers to gain access to cultural norms and attitudes that are often too complex for confirmatory, quantitative enquiry. As such, sample size is often very small in comparison to quantitative studies because qualitative research focuses on 
the depth of understanding cultural knowledge, rather than how many or what kinds of people hold the knowledge. McCracken (1988, p. 17) summarises this methodological paradigm perfectly: "...qualitative research does not survey the terrain, it mines it. It is, in other words, much more intensive than extensive in its objectives".

A PICO-based literature review (Hauser and Jackson, 2016) was conducted to establish current knowledge about the non-clinical benefits of EBVM and it concluded that while there are some papers suggesting a link between the practice of EBVM and better non-clinical benefits such as client satisfaction and client retention, a single study, focusing on the non-clinical benefits of EBVM, had yet to be produced.

With the limited knowledge generated from this activity, this study was designed to gain further understanding of the non-clinical benefits of EBVM. As recommended by Yin (2014), an interview guide containing broad, open-ended questions was developed to explore four key themes that emerged from the sparse existing knowledge: the definition of EBVM, the benefits of EBVM, the barriers to the adoption of EBVM and ideas for exploring the broader adoption of EBVM.

This study forms the first phase of a two-part, mixed-method research project that will establish the nonclinical benefits of EBVM. This first phase is a qualitative enquiry essential for determining the under-studied area of the non-clinical benefits of evidence-based medicine. Zikmund (2003) emphasises that this first, exploratory phase of a mixed-method project is particularly important when addressing under-studied areas of research as the discovery of new ideas is paramount in developing a well-informed confirmatory study.

The secondary aim of this research was to use the results of the interviews to create a survey that will empirically test the tacit knowledge documented in this paper. As such it was important that the interview participants were experienced supporters of EBVM and were able to share their intimate knowledge about this way of practicing veterinary medicine. With this in mind, the interview participants were recruited from the list of chairs of the RCVS Knowledge Groups. Chairs of RVCS Knowledge Groups are recognised leaders in the field of EBVM and hold these invited positions within the UK's Royal College of Veterinary Surgeons. Bogner, Littig and Menz (2009) discuss the virtues of conducting expert interviews in exploratory research. These authors suggest that efficiencies in time and knowledge are gained when qualitative data are collected from experts as they share a common background and relevance of the topic with the researcher so no time is lost with superfluous background explanations.

\section{METHODS \& MATERIALS}

As one of the aims of this research was to use the results of the interviews to create a survey for the second part of this study, it was important that the interview participants were champions of EBVM and were able to share their success stories about this way of practicing veterinary medicine. With this in mind, the interview participants were recruited from the list of chairs of the RCVS Knowledge Groups.

The interview participants were approached via e-mail which explained the purpose of the project and the ethics of the data collection process (i.e. participation in the research is entirely voluntary, despite the interviews being audio recorded all data would be anonymised and anyone was free to withdraw from the research at any time without fear of retribution). Two pilot interviews were conducted prior to the formal data 
collection and yielded no requirements for the data collection instrument or the research setting to substantively change. Interviews were conducted in person and via Skype in February and March, 2016. The interviews were audio recorded and transcribed verbatim and subsequently analysed using NVivo 11 software.

Interviewees were asked about their views on financial, commercial and business impacts of EBVM on the individual business. While financial benefits only include monetary aspects such as profit and loss of a practice, business and commercial benefits include a wider spectrum of benefits such as client satisfaction, employee engagement and reputation. These benefits can have a major impact on the success of a business despite being difficult to measure. Wider economic benefits, that include potential impacts on other industries, such as increased revenue in the meat processing industry due to better veterinary practice, were outside the scope of this study.

The interview guide (available as a supplementary file), the information sheet about the project and the consent form to be signed by research participants was scrutinised and approved by the Royal Veterinary College's Clinical Research Ethical Review Board (Approval \#2015 1457).

RESULTS

With ethical approval from the Royal Veterinary College's Clinical Research Ethical Review Board (URN 2015 1457), a total of 16 interviews were conducted with people who are dedicated to the practice of veterinary medicine through the principles of EBVM. Participants were self-selecting as their primary reason for contributing to this research was their dedication, enthusiasm and experience of practicing EBVM. Table 1 provides a summary profile of the research participants in terms of their main area of veterinary focus, age, gender, year graduated and whether or not they are a registered veterinarian. A seventieth EBVM Knowledge Chair was approached by declined to participate in the data collection because he considered that the research questions were being asked too prematurely for the current state of EBVM in UK veterinary practice.

Table 1: An overview of interview participant characteristics

\begin{tabular}{|c|l|c|c|c|c|}
\hline Interview & Main focus & Age & Gender & Graduated & Veterinarian \\
\hline 1 & Farm animal vet \& in academia & 55 & $\mathrm{M}$ & 1985 & Yes \\
\hline 2 & Small animal and exotics vet & 48 & $\mathrm{M}$ & 1990 & Yes \\
\hline 3 & Lab animal vet & 60 & $\mathrm{~F}$ & 1983 & Yes \\
\hline 4 & Animal nutritionist \& in academia & $>50$ & $\mathrm{~F}$ & DNA* & No \\
\hline 5 & Small animal vet in mixed practice & 64 & $\mathrm{~F}$ & 1975 & Yes \\
\hline 6 & Small animal vet \& in academia & 44 & $\mathrm{~F}$ & 1994 & Yes \\
\hline 7 & Equine and mixed practice & 42 & $\mathrm{M}$ & 1998 & Yes \\
\hline 8 & Mixed practice \& in academia & 50 & $\mathrm{M}$ & 1990 & Yes \\
\hline 9 & Equine & 59 & $\mathrm{M}$ & 1983 & Yes \\
\hline 10 & Farm Animal Vet \& in academia & 32 & $\mathrm{M}$ & 2006 & Yes \\
\hline 11 & Small animal \& in academia & 26 & $\mathrm{M}$ & 2013 & Yes \\
\hline 12 & Equine (but working for an NGO) & 34 & $\mathrm{~F}$ & 2007 & Yes \\
\hline 13 & Vet tech for small animals \& in academia & 40 & $\mathrm{M}$ & 2000 & No \\
\hline 14 & Small animal & 57 & $\mathrm{M}$ & 1982 & Yes \\
\hline 15 & Small animal & 43 & $\mathrm{M}$ & 1997 & Yes \\
\hline 16 & Equine & 58 & $\mathrm{M}$ & 1980 & Yes \\
\hline
\end{tabular}

*Did not answer

Table 1: An overview of interview participant characteristics 
Almost all participants were veterinarians, however, the interviews also included a veterinary technician and an animal nutritionist. Interviewees have a great range of focus and experience in veterinary medicine. Seven participants had some link to academia, while other participants worked in private practice, corporate practice, a government agency, or an NGO. While participants were purposefully not selected to be representative of the industry, it was ensured that the interviews were conducted with representatives from different fields of work, including: small animal, farm animal, equine, lab animal and mixed practices (which included exotics).

The following is a thematic analysis of the interview data. Many qualitative researchers follow the classic advice of Glaser and Strauss (1967) and take a grounded theory approach by allowing themes to naturally emerge from the data. Due to the specific nature of this research, we have been guided by the advice of Miles and Huberman (1994) who advocate using a 'start list' for generating themes. The 'start list' for this research was based on the research questions which were, of course, developed from existing literature (discussed in the Introduction to this paper). As such, the 'start list' was a loose list of themes that were grounded in the research questions.

The ethos and presentation of qualitative research is often criticised as being unsystematic, impressionistic, sloppy and unsophisticated (Glaser and Strauss, 1967). Similarly, Creswell (2009) discusses the erroneous sentiment of 'generalisability' of qualitative research. He goes to some lengths to explain that the virtue of qualitative research lies in the multidimensional description and development of themes in the specific context of the research setting and how particularity, rather than generalisability is the hallmark of qualitative research.

For presenting the results of the thematic analysis, we look to the advice of McCracken (1988) who discusses the method of inquiry associated with long interviews. He talks about the rich and useful knowledge that can be gained from the researcher providing sample quotes to capture the ideas and concerns of participants. The overall aim of this reporting method is for the abundant data to speak to the reader and provide a guided tour of the participants' thoughts and opinions on each theme.

\section{Defining EBVM}

In order to properly set the scene of EBVM for the research participants, it was necessary to first determine their understanding of the concept so participants were asked to define EBVM. Participants generally concurred that EBVM is "practicing medicine in a way that is supported by published evidence" (Participant 11) and is an effort for practitioners to separate themselves from practicing veterinary medicine on the way they were taught as opposed to making clinical decisions based on high-quality published data and published evidence. Support for these conclusions comes from the following participants:

- "Clinical practice that is based on current research and experience of, the pooled experience of many clinicians, that has been validated to be a good way of doing things..." (Participant 5)

- "...plying my craft or my trade in making my treatment decisions based upon the evidence that I have in front of me. All of the evidence, just not my personal opinion." (Participant 7)

While most participants were clear about the definition of EBVM, some participants extended the definition to include ensuring that the quality of evidence was sufficient to make good clinical decisions: 
- "It's just how you evaluate and choose your evidence.../...to actually structure and to formalise, to establish protocols for how you evaluate the information in front of you." (Participant 2)

- "...different scales of evidence, which might be, we've got very few meta-analyses in veterinary practice, but it might be published literature in your discipline or related disciplines." (Participant 10).

\section{Non-clinical benefits of EBVM}

Discussing the non-clinical benefits of EBVM initially proved difficult for participants as their immediate response was that the adoption of EBVM results in clinical success: it leads to an improved standard of care. Deeper questioning lead to further discussion about client satisfaction, client retention, improved reputation, confidence and a feel-good factor amongst practitioners.

The best evidence about the commercial benefits for EBVM that emerged from the data was from Participant 15 who said: "it's demonstrated that you know we're seeing increased sales of those things through the use of evidence based medicine". Participants largely agreed that practising EBVM will give a veterinary practice competitive advantage:

- "But what you can't get from anywhere else, are science and ethics. And if veterinary medicine can say, okay we're going to be scientific and practice evidence and we're going to be ethical, we then stand out." (Participant 9)

- "You can sustain a competitive advantage compared to your rivals because you're staying in touch and you know what works best, if it doesn't work best you remove it from your practice and replace it with something else that you have the evidence.../... when you are using evidence-based it gives you value, it's valued by the customer, it's rare because they don't use the same stuff you use, and at the same time it's inimitable which means it cannot be copied by [competitors] because they are not using the same approach as you are using." (Participant 1 ).

\section{Client satisfaction}

An improvement in client satisfaction was clear from a number of perspectives:

- Saving the client money "it can save your client money because if you're not trying different things to see what works and you're using what has been shown to work there's potentially a knock on financial benefit there, unless it's something expensive." (Participant 11);

- The perception of value held by the client "I would say input-output-outcome. This is the outcome that is valued by the customers, by clients, and evidence-based research studies is valued better by clients because they see it's working and it works in any circumstances." (Participant 1);

- Trust "I think the trust building aspect of it is really, really important." (Participant 9) and "People choose a vet because they get on with them, because a relationship is formed, because the trust, the bond formed between them and the practice." (Participant 2);

- Client appreciation "I think people generally appreciate that because it shows that you're being thorough" (Participant 10), "Based on the evidence and as soon as [the client is] invested and they are part of the treatment choice, ultimately everybody gets happier." (Participant 7) and "that client not only [is] receptive to the veterinarian looking into their animals specifically in disease, but really appreciate, you know, it is, it's extra work, it's extra time, it's a more personal touch." (Participant 13), 
- Over-coming the unknown "the best thing that I did and [clients] are so much more grateful, people respond so much better when you tell them I don't know, you know I don't know, I'm going to look it up, or we don't know, these are the options and this is why these are the options." (Participant 12) and "potentially more damaging to kind of trust with relationships with clients, and to be honest, people don't believe you that you've got all the answers all the time without having to go and look it up and that sort of thing." (Participant 10).

\section{Client retention}

While the ideas of client satisfaction and client retention are closely linked in the data, there was sufficient discussion to separate these as disparate concepts: client satisfaction being a short-term yet positive outcome from the provision of good clinical practice while client retention is focused on the longer-term positive externality of a trusting relationship being developed between client and veterinarian whereby the veterinarian is more than a valued service provider. The veterinarian takes on a more sophisticated relationship than an expert for hire and assumes the role of the client's trusted partner in providing long-term veterinary care:

- "You know from a very basic level, the longer your patient is away then the healthier they are, the more bonded your clients become and the more likely they are to come back and spend money with you. So you know from that perspective, there's a longevity to the relationship that you can form with the patients and the clients, so if you can keep animals healthy and provide them with the best possible care." (Participant 15)

- "I think that the main benefits are number one from a client standpoint is, it engenders a lot of trust, and if they understand where you're coming from, and they don't, they don't feel like they're being targeted and they feel like you're working in their best interests and the horse's best interests, I really do think that builds a strong loyal client base.../...Well how about practising evidence based medicine to try to do the right thing? That's when people will continue to come back to you. That's what I think." (Participant 9).

\section{Reputation}

Another closely-related issue to client satisfaction and retention is reputation. This emerged as a key theme from the data, which presented itself in a number of different ways:

- Approach to veterinary medicine "But the first opinion people coming through the door, why do they choose us over the other four practices in town? Because they like what they've heard about the reputation, they like the way we work, not especially what we do." (Participant 2)

- Trusted clinical practice "I know that [my vet] is evidence based, I know she reads the literature, I know she talks to her peers, I know she practices well." (Participant 12)

- Reliable procedures "And now everything's questioned so I think from a business point of view to support your integrity you have to be able to do some form of clinical governance" (Participant 4)

- Intangible value "I'm not sure quite how I would actually physically measure that. I guess there's maybe some value in having a reputation for doing things well" (Participant 11). 


\section{Confidence}

Discussions on the increased standard of care attributed to EBVM developed into insights into client satisfaction, client retention and reputation but most unexpectedly a lot of evidence emerged about how vets' confidence was developed from the application of EBVM and how it was a valuable opportunity to stimulate engaging, challenging discussions with colleagues. In terms of the non-clinical benefits of EBVM discussed for this research, the confidence that EBVM builds amongst vets and the satisfaction they gain from practicing good medicine was by far the most discussed theme within the data.

Satisfaction was a key word often used: "I think, in terms of job satisfaction, you get a lot more out of it because you don't feel like you've gone to work and winged it...You feel like you're ...doing clinical research, you feel like you're contributing to the wider port of knowledge. You feel like you're actually - you're doing something to help improve the profession you work in so it's got a personal satisfaction element to it as well.../... And the process I think makes everyone a lot happier because, if you like, there's stuff to support what they're doing." (Participant 11); "I think it gives me individual satisfaction that you're hopefully doing a good job and you are providing the 'best', in inverted commas, service you can do under the circumstances." (Participant 16).

Employee engagement emerged as another key theme-based on the ideas that EBVM creates debate and develops an intellectual challenge for veterinary professionals who are motivated by constantly being challenged: "by getting people together and sharing the ideas and being collaborative about it, then you know then we've been able to drive enthusiasm amongst ourselves and the barriers can fall away really, once you do that." (Participant 15).; "When you do evidence based medicine you become more invested in what you're doing and when you become more invested in what you're doing, you become more passionate about what you're doing. So in an intangible way, I see evidence based medicine, just being a huge asset in terms of confidence and, ownership or passion in the field.../... mean from my own personal perspective, it has certainly invigorated my passion for the field." (Participant 13); "And another perk is to share the experience. If you do evidence-based [sic] you can share the experience and you can get the best experience in this regard." (Participant 8).

Clinical decision making and the confidence that comes with good decision making were key issues raised; as was the issue of minimising waste generated by bad decision making: "I think it gives more confidence in clinical decision making, which everybody wants, right?" (Participant 13); "[you] have more confidence in what you're doing...you've not got that internal doubt or panic of is what I'm doing the right thing to do because you always have that no matter how many times you do something." (Participant 11); "It's personal, more than anything else. I can actually go to bed with a hand on my heart and say, I applied the best evidence as far as I am aware, because ... the evidence that I have today, right now, means I can hand on heart, go to sleep and tell them I did the best possible job." (Participant 7).

The ability to deal with clients, particularly, difficult questions from clients emerged as an issue that built confidence: "...EBVM may form part of your defence" (Participant 2); "the client, they think they're so much better informed so they can access doctor Google, so I think you've got to be able to justify what you're doing in a constructive way, in a proven" (Participant 4); "I think when challenged about the reasons why we do things, then we are able to support the reason why we do things with evidence" (Participant 15).

\section{Barriers to the wider adoption of EBVM}


The two major barriers that were cited to the wider adoption of EBVM were time and resources. "I think the two big barriers are time, and access to evidence." (Participant 16) While participants recognised the long-term benefits of EBVM (e.g. developing staff knowledge and their confidence, knowing that clients are being provided with the best care for their animal(s)), there was much discussion around the idea that EBVM is a long-game that is resource-intensive and time-consuming in the short term.

Time was discussed in terms of the time required to investigate the evidence based for individual cases:

- "The ability to understand and interpret research findings." (Participant 12);

- "I think time is the big issue in practice, because you're just so busy all the time, to sit down and actually look at journals, it's really difficult." (Participant 6).

Discussing the evidence with colleagues was discussed in a positive light for sharing information and engaging with like-minded people but it did also involve taking time away from clinical practice: "I think the time is probably the next biggest barrier. People are overstretched and overworked and feel that it's just yet another thing that they have to do." (Participant 15).

There was an overwhelming response from participants that there is a paucity of publicly-available resources for truly adopting EBVM in practice. Upon digging deeper into this theme, there is evidence to suggest that access to literature may not actually be the true problem. The problem may actually lie in vets' readiness and willingness to access the literature in that actually sourcing literature to make clinical decisions is not at the top of their minds:

- "I think the biggest barrier is probably access to evidence" (Participant 11)

- "...one of the problems is in veterinary medicine we don't have the huge number of resources that have been developed to support physicians." (Participant 3)

- "Possibly access to the materials as well, because I know that we tell the students here and I tell colleagues, you can go to the RCVS library and you can get access, and if you're a member of BSCVU you can get access, but I think there's still a bit of a barrier that a lot of people don't know, that they can actually access all these articles really easily." (Participant 6$)$.

"The industry culture is the enemy of innovation and it's the enemy of evidence-based research and studies" This evidence from Participant 1 summarises another theme that emerged from the data about the barriers to adopting EBVM. To start with, some participants discussed their perceptions of the profession's current reliance on 'eminence rather than evidence':

- "Most of veterinary medicine that I see has no evidence base, and is detrimental to animal welfare, doesn't work, and that is very difficult, so I think there's a huge barrier" (Participant 12)

- "I think there's a lot of pride that goes with veterinary medicine with practising medicine.../...Anecdotal evidence is extremely powerful, so having a case that you treated one particular way and it seemed to 
work, you know it's extremely powerful and it can be very difficult to overcome that, if it's not evidence based practice." (Participant 15)

- " "[EBVM] never occurred to me as a new grad, I just believed everything." (Participant 6).

There also appears to be a problem with the quality of evidence available to practitioners in terms of depth of coverage, validity of information and quality of information:

- "The evidence base in veterinary medicine is so weak and patchy, that it's very, very difficult to practice anything approaching what Doctor Greenhalgh or Cochrane would recognise as evidence based medicine." (Participant 14)

- "A little knowledge is dangerous" (Participant 11)

- "But certainly in the veterinary field there is a huge lack of evidence, and a lot of what is written is clinically irrelevant or only marginally relevant so the quality of the stuff that's out there is always going to be a challenge." (Participant 14).

An issue associated with industry culture is the lack of awareness by clients, their lack of demand for EBVM and some owners' demand for 'quick-fix, low-cost solutions':

- "So you have to educate clients, number one. About the true cost of healthcare." (Participant 7)

- "I think for most owners and most practitioners, both side of the patient on the veterinary client patient transaction, I think it's more important for them to have something to do, than it is to do something that's effective." (Participant 9)

- "...the challenge is getting the public to come with us really." (Participant 15).

The final issue that emerged from the data about barriers to the adoption of EBVM is the attitudes of educational institutions. The foremost issue here related to veterinary schools' lack of attention to EBVM but the issue of teaching and assessing these skills was also raised:

- "So eminence based medicine is the biggest barrier to evidence based medicine. And that's deeply, deeply rooted in the vet schools." (Participant 14)

- "I think it's a cultural educational issue, first and foremost, but assuming we have the attitude to practice EBVM, then it comes down to time and access." (Participant 13)

- "And then the next big barrier I guess is the skills gap. It's you know not feeling that you have the skills necessary to appraise evidence and to, you know to formulate the right sort of questions and to put together, you know the, the information needed in a format that's, you know that's acceptable." (Participant 15).

4. Increasing the adoption of EBVM 
The two most prominent issues to emerge from the data about increasing the adoption of EBVM are that 1) EBVM needs management support: journal clubs, forums, training opportunities, CPD, time for reading, all members of the vet team need to be included, senior vets as role models, vet techs/nurses need to be involved in EBVM (not just vets) and 2) EBVM needs investment: funded research projects, support services, journal access.

In terms of management support for EBVM, the interview data showed support for the heightened level of employee engagement that came about from vets and vet nurses who are given the time and intellectual freedom to practice EBVM rather than being trapped under a heavy case load. Further evidence is provided by these quotes:

- "I think businesses would definitely benefit if they were to allow, you know, you could just like one afternoon a week or every month or something tell people to go and spend time reading or looking up things. I think that would be a really good move because I think they would see a definite improvement in terms of staff performance and turnover.../...I think management generally need to be more accepting of EBVM, of what it can do for their business in terms of improved client satisfaction, improved turnover, improved handling of client complaints and things like that." (Participant 11)

- "...we kind of embark on the journey together.../... it's been a pleasure to work through the challenges together really." (Participant 15)

- "I think that [good medicine, good science and good ethics] go hand in hand, and I think that the important thing, I think that EBVM can be a cornerstone of that, and should be a cornerstone of that, but I just think that, like I said change has to come top, from top down." (Participant 9)

- I'm a huge proponent for the role of veterinary nurse, veterinary technician in this process and I think EBVM can only be accomplished as a team." (Participant 13)

As for increased investment in EBVM, the following data showed demand for opportunities to teach practitioners about considering and understanding high-quality evidence, how to discuss high-quality evidence with important stakeholders and that using EBVM is easier over time as it becomes habit:

- "I think more CPD events on how to perform EBVM would be quite useful. I think the knowledge[EBVM learning] - the learning resource that they've published is really good and I think they just need to work and make sure as many vets know about that as possible because it is a really good open source of information on how to EBVM." (Participant 11)

- "The development of the RCVS knowledge and the introduction of the grants for, for supporting EBVM, were probably a turning point for us as a business and I guess my activity, with regards to EBVM has increased significant learning since then." (Participant 15).

Participants 3 and 12, respectively, commented on the need for open-access publications: "So this is where we get into open publication, the whole concept of open access science, and I think that's another barrier, or another way to break down the barriers would be to have more open access." and "I think scientific publishing is going to have to change. It's going to, I mean it's changing already right with open access, and you know more and more is open access, more and more is free" which is linked to comments made by Participant 2: "It's 
there, but you know you want to bring people together, and I think that's probably the key thing, is that if we find to make bridging university, like academia and practice gap, people will start seeing more relevant meaning to it.../...The way barriers can be overcome fundamentally is communication and not protectionism."; "I think that there needs to be some acknowledgment, there might need to be some financial support or expertise support that go into groups and practices and help them do that. I think it's unrealistic to expect practitioners to do that." (Participant 4)

Participant 5 provided some constructive comments to improve the adoption of EBVM: "spread it out a bit, I mean having two major conferences a year at the moment. You should perhaps take it smaller, you've probably done a bit of this I think, perhaps going out to local veterinary societies, but maybe even contact larger practices in an area where they've perhaps got, you know we've got a room downstairs, our waiting room we use as a meeting room sometimes for the vets from other practices........Somebody from Nottingham or from the university, committee of EBVM should come and perhaps, talk about it a bit more, so that everybody knows about it, the nurses and everybody as well.". Participant 4 provided another interesting idea: "Well I tell you what the college needs to do, they need to buy into the fact that you can do a residency in practice, because this would fit a residency so well. That's what they need to do. It doesn't need to be hospital-based or university-based. It can be in practice."

DISCUSSION

This research yielded themes that are of considerable importance to the veterinary professions at present and that practicing EBVM may derive greater benefits than superior clinical outcomes. McCracken (2013) suggested that financial and operational efficiencies are outcomes of evidence based medicine (EBM) in the human world but these did not emerge as themes from the data. More in-keeping with the logic of Caldwell (2001) who advocates the convergence of EBM healthcare systems objectives, we found that practicing EBVM is not restricted to strong clinical practice but is also related to greater client satisfaction in the short term, and to long-term client retention. However, it was also highlighted that often clients are not aware of EBVM or prefer a 'quick fix' solution. Another closely-related issue to client satisfaction and retention is reputation. This emerged as a key theme from the data, which presented itself in a number of different ways including the way a better reputation is gained by a different approach to veterinary medicine and performing reliable procedures. These themes essentially link to professionalism and professional identity. One of the key constructs of professionalism is the professional's ability to communicate. Previous studies have discussed the importance of communicating with clients to develop sustainable business practice (Backynsky et al., 2013) and managing client expectations (Mossop et al., 2015). The present research adds to this body of knowledge by providing EBVM as another tool for enhancing client satisfaction and enabling client retention.

By far the most discussed theme that emerged from the data, was how EBVM influences vets' confidence and the satisfaction they gain from practicing good medicine. The practice of EBVM was seen as a valuable opportunity to stimulate engaging, challenging discussions with colleagues. Similarly, employee engagement emerged as another important theme based on the ideas that EBVM creates debate and develops an intellectual challenge for veterinary professionals. This is a particularly important finding of the research due to the current climate of the UK veterinary professions. The Royal College of Veterinary Surgeons and the British Veterinary Association have collaborated in the Vet Futures initiative (http://www.vetfutures.org.uk/). Research findings of this initiative have outlined several key ambitions and recommendations for the profession, two of which are: 1) "confident, resilient, healthy and well-supported [professionals]" and 2) "a broad range of diverse and rewarding career paths" (Vet Futures, 2015, p. 4). The present research demonstrates that practicing of EBVM could be one of the avenues the profession could pursue in its efforts to ensure its people are well-supported and work in interesting, developing careers. Similarly, VN Futures (2016) 
discusses some of the causal factors of stress in the veterinary professions and cites "professional isolation" as one of the reasons why people in the veterinary professions are so stressed. Once again, the social and developmental aspects of practicing EBVM that emerged from the present research could offer an important opportunity for relieving this pressure point.

A theme to recently emerge from the veterinary professionalism literature is professional identity. The development of professional identity starts with the understanding of professionalism and under-lying foundations of professionalism are the ability to balance multiple responsibilities and manage professional challenges (Armitage-Chan, Maddison \& May, 2015). Balancing multiple responsibilities involves engaging with different parties both internal and external to the veterinary environment who, managing professional challenges, relates to controlling work/life balance and responses to difficult or emotional clients. Evidence from the present research suggests that vets' ability to deal with the increasing expectations of clients, achieving goals through team performance, and gaining confidence from practicing good veterinary medicine could be yet another benefit of EBVM to the audience interested in professional identity formation.

One of the main limitations of this study was that there were a number of important general issues that also emerged about the non-clinical benefits of EBVM that did not suitably fit into the results of the thematic analysis of interview data. One of the RCVS Knowledge Chairs who was invited to participate in this research declined because he considered that it was far too early to attempt to quantify the non-clinical benefits of EBVM; similarly, one participant could not see a direct link between practising EBVM and a direct business advantage.

A further limitation was that the increased standard of care was the most intuitive response participants gave when discussing EBVM, and it was at times challenging to move the discussion to the non-clinical benefits. However, through further questioning far more detailed themes emerged about clients, practice reputation and staff confidence.

We take a pragmatic philosophical stand point to this research and acknowledge that this exploration of expert opinions of EBVM is not where this enquiry terminates. This qualitative phase of the research has allowed for the rich and in-depth exploration of key stakeholder perceptions on which we can now use as a platform for confirmation and explanation of the non-clinical benefits of EBVM in a survey of the professions; that is, empirically testing the theories that were grounded in our research questions. This pragmatic, pluralist approach is best described as mixed-method research (Johnson \& Onwuegbuzie, 2004) which rejects Howe's (1988) incompatibility thesis that the quantitative and qualitative methodologies can never be combined. In summary, this study forms the first phase of a two-part, mixed-method research to establish the non-clinical benefits of EBVM. From the findings of this explorative study a survey will be created that will empirically test the knowledge documented in this paper.

CONFLICT OF INTEREST

Acknowledgments: We would like to thank RCVS Knowledge for its generous support of this project, our Coinvestigator: Graham Milligan MRCVS and all the interview and survey participants

Funding: RCVS Knowledge grants.

Competing interests: There are no areas of conflicting interest associated with this work. 
1. Armitage-Chan, E. Maddison, J. \& May, S. A. (2016) What is the veterinary professional identity? Preliminary findings from web-based continuing professional development in veterinary professionalism. Veterinary Record, 178 (13), p. 318. http://dx.doi.org/10.1136/vr.103471

2. Bachynsky, E. A. et al. (2013) A survey of the opinions of recent veterinary graduates and employers regarding early career business skills. Veterinary Record, 172 (23), p.

604. http://dx.doi.org/10.1136/vr.101376

3. Bogner, A. Littig, B. \& Menz, W. (2009) Introduction: Expert interviews - An introduction to a new methodological debate in Interviewing Experts, Palgrave Macmillan: UK.

4. Caldwell, C. (2001) The business case for evidence-based medicine. International Journal of Medical Marketing, 2 (1), pp. 54-72. http://dx.doi.org/10.1057/palgrave.jmm.5040055

5. Creswell J.W. (2009) Research Design: Qualitative, Quantitative and Mixed Methods Approaches. $3^{\text {rd }}$ Sage Publications: California, USA.

6. Glaser B.G. \& Strauss A.L. (1967) The Discovery of Grounded Research: Strategies for Qualitative Research. Aldine De Gruyter: New York, USA.

7. Hauser, S. \& Jackson E.L. (2016) Non-Clinical Benefits of Evidence-Based Veterinary Medicine.Veterinary Evidence, [S.I.], 1(3), aug. 2016. ISSN 2396-9776. Available at: $<$ https://veterinaryevidence.org/index.php/ve/article/view/34> [Accessed: 27 March2017].

8. Henry, C. \& Jackson, E.L. (2015) 'Women's entrepreneurship and the future of the veterinary profession', e-Organisations and People, 22 (3), pp. 34-42.

9. Howe, K. R. (1988) Against the quantitative-qualitative incompatibility thesis or dogmas die hard. Educational Researcher, 17 (8), pp. 10-16. http://dx.doi.org/10.3102/0013189X017008010

10. Johnson, R. B. \& Onwuegbuzie, A. J. (2004) Mixed methods research: A research paradigm whose time has come. Educational Researcher, 33 (7), pp. 14-26. http://dx.doi.org/10.3102/0013189X033007014

11. McCracken, B. (2013) Building a business case for evidence-based medicine, ClinicalKey, Elsevier.

12. McCracken, G. (1988) The Long Interview, Qualitative Research Methods Series 13, SAGE Publications: California, USA.

13. Miles M.B. \& Huberman M. (1994) Qualitative Data Analysis: A Sourcebook of New Methods, Sage Publications: California, USA.

14. Mossop, L. et al. (2015) Communication skills training: what the vet schools are doing. Veterinary Record, 176 (5), pp. 114-117. http://dx.doi.org/10.1136/vr.h425

15. Vet Futures (2015) Taking Charge of our Future: A vision for the veterinary profession for 2030 , Available at: http://www.vetfutures.org.uk/resource/vet-futures-report/ [Accessed: 27 March2017].

16. VNFutures (2016) VNFutures - Taking charge of our future together. Available at: http://www.vetfutures.org.uk/resource/vn-futures-report-and-action-plan/ [Accessed: 27 March2017].

17. R.K. (2014) Case Study Research Design and Methods. 5th ed. Sage: Thousand Oaks, USA.

18. Zikmund, W.G. (2003) Business Research Methods. $7^{\text {th }}$ Thomson South-Western: Ohio, USA. 


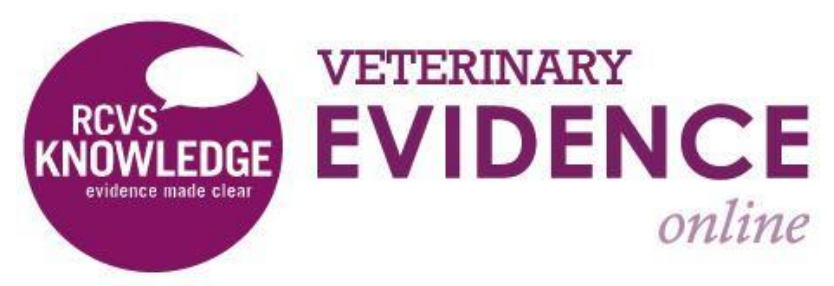

\begin{abstract}
Intellectual Property Rights
Authors of Articles submitted to RCVS Knowledge for publication will retain copyright in their work, but will be required to grant to RCVS Knowledge an exclusive license of the rights of copyright in the materials including but not limited to the right to publish, re-publish, transmit, sell, distribute and otherwise use the materials in all languages and all media throughout the world, and to license or permit others to do
\end{abstract} so.

Authors will be required to complete a license for publication form, and will in return retain certain rights as detailed on the form.

Veterinary Evidence and EBVM Network are RCVS Knowledge initiatives. For more information please contact us at editor@veterinaryevidence.org.

RCVS Knowledge is the independent charity associated with the Royal College of Veterinary Surgeons (RCVS). Our ambition is to become a global intermediary for evidence based veterinary knowledge by providing access to information that is of immediate value to practicing veterinary professionals and directly contributes to evidence based clinical decision-making.

www.veterinaryevidence.org

RCVS Knowledge is a registered Charity No. 230886. Registered as a Company limited by guarantee in England and Wales No. 598443.

Registered Office:

Belgravia House

62-64 Horseferry Road London SW1P 2AF

This work is licensed under a Creative Commons Attribution 4.0 International License. 